\title{
CONFLITO EM REDES: ORIGENS E DESTINOS COTADOS
}

\author{
Hilton Vieira Machado \\ Universidade de Brasília - UnB \\ Brasília - DF \\ est@unb.br
}

\author{
Rogério Lopes Sinotti * \\ Exército Brasileiro e \\ Universidade de Brasília - UnB \\ Brasília - DF \\ sinotti@unb.br \\ * Corresponding author/autor para quem as correspondências devem ser encaminhadas \\ Recebido em 06/2003; aceito em 03/2004 após 2 revisões \\ Received June 2003; accepted March 2004 after two revisions
}

\begin{abstract}
Resumo
Num confronto, evasor e detector buscam estratégias ótimas sobre, respectivamente, as rotas origemdestino e os arcos de possível bloqueio (com probabilidades de detecção conhecidas) num jogo matricial de soma zero avaliado pela probabilidade média de detecção (Washburn \& Wood, 1995). Múltiplas aplicações civis e militares continuam estimulando pesquisa na área (Caulkins et al., 1993; Israeli, 1999; Bell, 2003). Quando há várias origens e destinos, com cotas pré-fixadas (totais percentuais de fluxo), a redução dimensional do problema do evasor (no par dual) a um problema padrão de fluxo máximo, não parece diretamente possível nem analiticamente acessível. Propomos aqui uma abordagem indireta, por delimitação sucessiva do fluxo, num algoritmo exato. Testes preliminares com redes de dimensão reduzida e simulações múltiplas com uma rede de porte médio da literatura têm indicado bom desempenho computacional.
\end{abstract}

Palavras-chave: redes cotadas; jogos de detecção e evasão; redução computacional.

\begin{abstract}
In a network confrontation, evader and detector look for optimal strategies on origin-to-destination trajectories and blocking arcs (with assigned detection probabilities) in a zero-sum matrix game with the mean capture probability as pay-off (Washburn \& Wood, 1995). Related active research goes on, stimulated by multiple civil and military applications (Caulkins et al., 1993; Israeli, 1999; Bell, 2003). For multiple origins and destinations with pre-established quotas, the dimensional reduction of the evader problem (in the dual pair) to a standard maximum flow problem doesn't seem directly possible or analytically amenable. Herein an indirect approach, based on successive delimitations of the flow, is proposed, leading to an exact algorithm. Preliminary tests with limited-size problems and multiple simulations on a medium-size network of the literature have shown good computational performance.
\end{abstract}

Keywords: quotas on networks; detection and evasion games; computational reduction. 


\section{Introdução}

A localização estratégica de recursos escassos ao longo de uma rede, visando detecção (interceptação, bloqueio ou apenas supervisão) de tráfego indesejável (inimigo, ilícito, clandestino) controlado por agentes racionais é por natureza, um problema de Jogos. Em sua forma mais simples, e por isso mesmo mais aplicada, trata-se do conflito de interesses no confronto entre dois jogadores: o evasor, que escolhe a rota a ser utilizada (para fuga, tráfico, transporte de suprimentos, evasão de capital) e o detector, que, independentemente, seleciona um arco da rede para se instalar (patrulhar, interceptar, inspecionar).

Os primeiros trabalhos sobre Interdição em Redes datam dos anos 50, resultado a posteriori do esforço de equipes multidisciplinares durante a $2^{\mathrm{a}}$ Guerra Mundial, destacando-se nos Estados Unidos, o Grupo de Avaliação Operacional do Departamento Naval (sobre o assunto, ver Danskin, 1962). Nas décadas de 60 e 70, diversos modelos foram propostos e intensamente analisados, entre outras, pela necessidade de bloquear rotas de suprimento da guerrilha no Vietnã (Wolmer, 1964; Durbin, 1966; Wolmer, 1970a e 1970b; McMasters \& Mustin, 1970), muitos deles utilizando como ferramenta teórica e computacional o algoritmo de Ford e Fulkerson, de 1954, sobre fluxo máximo e corte mínimo em redes capacitadas. Mais recentemente, Washburn (1980 e 1983), Steinrauf (1991), Wood (1993) e Caulkins et al. (1993) entre outros, abordaram modelos ainda determinísticos, de busca, obstrução simultânea de arcos e nós, etc., assim como, aplicações. O trabalho supracitado de Steinrauf, por exemplo, envolvia a apreensão de precursores químicos numa rede rodo-fluvial no Rio Mamoré na fronteira Brasil-Bolívia.

Em 1998, Cormican et al. analisaram uma versão estocástica, com solução à Stackelberg, isto é, em que obstrução - destruição, parcial ou não, dos arcos da rede, é feita preliminarmente pelo interditor, o adversário procurando então otimizar seu uso - fluxo máximo, custo mínimo, etc.

Em 1999 Israeli (a) reviu a literatura de Interdição, (b) estendeu os modelos a sistemas gerais, em princípio lineares, mas não necessariamente ligados a redes, designados Linear System-Interdiction Problems (LSIP) e Linear System-Defense Problems (LSDP) pelo autor (interdição e defesa), (c) propôs alguns algoritmos e heurísticas para a redução aproximada de problemas específicos em malhas $\mathfrak{R}$ e (d) relatou testes numéricos com malhas de porte médio, envolvendo fluxo máximo ou caminho mínimo como objetivo na otimização.

Trata-se de problemas estruturados, do tipo $\min _{x} \max _{y} c^{\prime} y$ e $\max _{w}\left(\min _{x} \max _{y} c^{\prime} y\right)$ em que as variáveis de decisão $\boldsymbol{w}, \boldsymbol{x}$ e $\boldsymbol{y}$, em $R^{|K|}$ (as duas primeiras binárias), representam respectivamente o reforço e o ataque seletivos ao conjunto $K$ de arcos de $\mathfrak{R}$, e seu nível de utilização na rede remanescente (após sua destruição parcial). O modelo básico assume limitações lineares de recursos, $\mathbb{H} \boldsymbol{w} \leq \boldsymbol{h}$ e $\mathbb{T} \boldsymbol{x} \leq \boldsymbol{t}$, com $0 \leq x_{k} \leq 1-w_{k}$, isto é, arcos reforçados não são atacados e $0 \leq y_{k} \leq c_{k}\left(1-x_{k}\right)$, onde $c_{k}$ é a capacidade original do arco $k$. Trata-se portanto de jogos à Stackelberg: no primeiro, o atacante considera a melhor utilização a posteriori da rede pelo usuário; no outro o usuário planeja reforçar $\Re$ de modo a reduzir as conseqüências de um ataque bem executado, o pior possível, de seu ponto de vista.

Nos testes, Israeli gera randomicamente instâncias (10) de malhas (com cerca de 100 nós) e utiliza diversas técnicas (decomposição, cortes, propostas heurísticas e híbridas) para o cálculo aproximado dos ótimos. Apesar de tolerâncias da ordem de 3600 segundos de CPU, 
os resultados nem sempre se mostraram bem definidos (discriminação quanto à eficiência relativa) e, conforme se poderia esperar, exibiram grande sensibilidade à ordem de grandeza dos dados.

No trabalho, Israeli comenta que, além da possibilidade das aplicações mais óbvias, militares (detecção ou condução de ataques, defesa de rotas críticas, deslocamento de material e contingentes, questões de suprimento) e civis (tráfico em suas diversas formas, contrabando comercial, de capital, imigração) outras têm sido seriamente cogitadas, destacando-se a criação, em 1996, da comissão presidencial americana PCCIP (White House Executive Order 13010, 07/15) visando a proteção e sobrevivência de infraestruturas críticas, consideradas vitais, cuja incapacitação poderia vir a debilitar a segurança econômica e a defesa do país, incluindo telecomunicações, energia, redes financeiras, transportes, água, etc, a serem protegidos de possíveis ataques físicos ou eletrônicos.

A grande maioria dos modelos de interdição em redes era determinística até 1995 , quando Washburn \& Wood propuseram para o modelo estocástico básico, a redução do jogo a um par dual fluxo máximo-corte mínimo na rede, uma substancial vantagem computacional que evita a necessidade da enumeração a priori das rotas ao longo da rede (no jogo, as estratégias puras do evasor), utilizando o algoritmo de Ford \& Fulkerson (1954).

Mais recentemente, algumas extensões do modelo Washburn-Wood, algoritmos e testes foram desenvolvidos em Machado \& Sinotti (2002) (ver também Lima \& Muniz, 2002). No presente trabalho buscamos resolver o caso em que são estipuladas, nos extremos da rede, cotas de utilização, mais precisamente, as freqüências de uso de cada uma das origens e de cada um dos destinos da rede $\mathfrak{R}$, no processo de evasão, uma extensão conceitualmente simples, mas que exige métodos indiretos para resolução via Ford e Fulkerson, e que é considerada relevante por suas possíveis aplicações - e.g., em tráfico ilícito em que se tem estimativas das quantidades relativas de produção e consumo nos diversos centros envolvidos. A Seção 2 introduz conceitos básicos e formaliza o problema como um jogo matricial de soma zero. O dilema da redução do problema do evasor a um problema de fluxo máximo, segundo o esquema Washburn-Wood, é discutido na Seção 3, em que se ressalta a dificuldade introduzida pelas cotas nos extremos da rede. A Seção 4 propõe um algoritmo "de delimitação sucessiva de fluxos" cuja convergência é estabelecida na Seção 5. Sua implementação é ilustrada na Seção 6, em uma rede simples (17 arcos, 23 rotas). A Seção 7 relata resultados de testes numéricos preliminares, considerados satisfatórios, envolvendo uma malha de porte médio (cerca de 58.000 rotas). Finalmente, a Seção 8 delineia algumas conclusões e propostas naturais para futura investigação.

\section{O Modelo - Conceitos e Notação Básicos}

Trabalhamos com uma rede dimensionada $\mathfrak{R}$, com várias origens e destinos $-\operatorname{arcos} k \in K$, nós $n \in N$, origens $i \in I$, destinos $j \in J$ (extremos $e \in E=I \cup J)$ e $\operatorname{rotas} l \in L$, de $I$ para $J$. O dimensionamento é descrito pelos vetores de $\operatorname{cotas} \boldsymbol{a}=\left(\alpha^{\prime}, \beta^{\prime}\right) \in R^{|K|}$, onde $\alpha$ e $\beta$ são distribuições de probabilidades sobre $I$ e $J$ respectivamente, e $\boldsymbol{p} \in R^{|K|}$, de probabilidades de detecção $p_{k}$, arco a arco.

As estratégias mistas dos jogadores são $\boldsymbol{x}$ e $\boldsymbol{y}$, distribuições sobre os $\operatorname{arcos} k$ e as rotas $l$ : freqüências de monitoramento de cada trecho (arco) $k$ da rede pelo detector e de uso da particular rota $l$ pelo evasor, isto é, das escolhas independentes, de $k$ e de $l$, por ocasião do 
confronto dos jogadores que buscam por decisões ótimas, individuais. Trata-se de um jogo matricial de soma zero (ver e.g., Davis, 1973) com um condicionamento particular sobre as estratégias do evasor; mais especificadamente, sobre as frequiências com que as diversas origens e destinos serão utilizados.

Introduzimos aqui duas matrizes binárias de incidência (um termo usualmente reservado para a relação vértice $\times$ arco, ver Boaventura Netto (1996) e Szwarcfiter (1984)):

$\mathbb{I}(\operatorname{arco} \times$ rota $)$ de ordem $|K| \times|L|, \mathbb{I}_{k, l}=1 \leftrightarrow k \in l$, isto é, arco ao longo da rota,

$\mathbb{R}($ extremo $\times$ rota $)$ de ordem $(|I|+|J|) \times|L|, \mathbb{R}_{e l}=1 \leftrightarrow e \in l$, extremo da rota.

Temos ainda os seguintes elementos auxiliares: $\mathbb{D}=\operatorname{diag}(\boldsymbol{p})$, a matriz diagonal de detecção (ordem $|K|$ ), o vetor de capacidades nos arcos, $c \in R^{|K|}$, com $c_{k}=1 / p_{k}$ ( $c_{k}$ um número suficientemente grande, se $\left.p_{k}=0\right)$, a matriz $\mathbb{C}=\operatorname{diag}(\boldsymbol{c})$ e o vetor $\boldsymbol{e}$, que chamaremos de vetor unidade, de componentes iguais a 1 ( $\boldsymbol{e}$ é a soma dos vetores unitários do espaço). A conveniência de introduzir o vetor $\boldsymbol{c}$, isto é, de considerar a inversão $c_{k}=1 / p_{k}$, ficará evidente quando mais tarde convertermos o jogo num par dual fluxo máximo-corte mínimo: o "tráfego fica congestionado" em arcos de baixa capacidade, isto é, de alta probabilidade de detecção, direcionando assim, indiretamente, a busca pela estratégia ótima de evasão.

As estratégias dos jogadores são descritas geometricamente pelos seguintes símplices:

$S_{L}=\left\{\boldsymbol{y} \in R^{|L|}: \boldsymbol{y} \geq 0, e^{\prime} \boldsymbol{y}=1\right\}$, o simplexo das estratégias, não cotadas (livres), do evasor.

$S_{\mathrm{L}}=\left\{\boldsymbol{y} \in S_{L}: \mathbb{R} \boldsymbol{y}=\boldsymbol{a}\right\}$, o conjunto das estratégias proporcionais do evasor, isto é, que respeitam as cotas $\alpha_{i}$ e $\beta_{j}$ nos extremos da rede. $S_{L}$ é um politopo em $S_{L}$, com, digamos, $T$ pontos extremos $\boldsymbol{v}_{1}, \boldsymbol{v}_{2}, \ldots, \boldsymbol{v}_{T}$. Observe que se $\mathbb{V}$ é a matriz $|L| \times T$ com colunas $\boldsymbol{v}_{t}$, e $S_{T}$ é o simplexo $\left\{\boldsymbol{z} \in R^{T}: \boldsymbol{z} \geq 0, e^{\prime} \boldsymbol{z}=1\right\}$, temos $\mathbb{S}_{\mathrm{L}}=\mathbb{V}\left(S_{T}\right)\left(\mathbb{S}_{\mathrm{L}}\right.$ é a envoltória convexa dos $\mathrm{T}$ vetores $\boldsymbol{v}_{t}$ ).

$S_{K}=\left\{x \in R^{|K|}: x \geq 0, e^{\prime} x=1\right\}$, o simplexo das estratégias do detector.

No confronto, descrito pelo par $(\boldsymbol{x}, \boldsymbol{y})$, utilizaremos como função de avaliação (ganho, pay-off) a probabilidade média de detecção:

$$
g(\boldsymbol{x}, \boldsymbol{y})=\boldsymbol{x}^{\prime} \mathbb{D} \mathbb{I} \boldsymbol{y}=\sum_{k \in l} p_{k} x_{k} y_{l}
$$

onde $\boldsymbol{x} \in S_{K}, \boldsymbol{y} \in \mathbb{S}_{\mathrm{L}}$ e o somatório é duplo: sobre todos os pares $k \in K, l \in L$, para os quais $k \in l$ - uma notação sintética usada deste ponto em diante. Trata-se portanto de um jogo matricial de soma zero, com problemas simétricos:
(a) $\max _{x} \min _{y} g$ (do detector)
e
(b) $\min _{y} \max _{x} g,($ do evasor) 
Como $\boldsymbol{y}$ não varia livremente no simplexo $S_{\mathrm{L}}$ e sim em $\mathcal{S}_{\mathrm{L}}$, um subconjunto de $S_{\mathrm{L}}$, a transformação clássica do jogo num par dual em Programação Linear (ver e.g., Gass, 1969) não se aplica diretamente. Entretanto, como $\mathbb{S}_{\mathrm{L}}=\mathbb{V}\left(S_{T}\right)$, podemos reescrever $g$ como

$$
g(\boldsymbol{x}, \boldsymbol{z})=\boldsymbol{x}^{\prime} \mathbb{D} \mathbb{I V} \boldsymbol{z} \quad \text { com } \quad \boldsymbol{x} \in S_{K}, \boldsymbol{z} \in S_{T} .
$$

Temos agora um jogo matricial na forma clássica, com matriz $\mathbb{M}=\mathbb{D} \mathbb{I} \mathbb{V}$ de ordem $|K| \times T$ e estratégias mistas $\boldsymbol{x}$ sobre os arcos $k$ (cada arco é uma estratégia pura do detector) e estratégias $\boldsymbol{z}$ sobre as estratégias proporcionais básicas $\boldsymbol{v}_{t}$ (as puras do evasor). É possível portanto (pelo menos em princípio) considerar a redução do problema, proposta por Washburn \& Wood (1995), à um par dual fluxo máximo × corte mínimo (Ford \& Fulkerson, 1954).

Denotaremos por $L(k), L(i), K(l), K(i)$, etc, o conjunto das rotas passando pelo arco $k$, iniciando na origem $i$, dos arcos ao longo da rota $l$, iniciando em $i$, etc, respectivamente. Assim, por exemplo, dizemos que, um subconjunto $C$ de $K$ é um corte em $\Re$ quando $L(C)=L$, isto é, toda rota passa por algum dos arcos $k \in C$ (a remoção de $C$ desconecta as origens dos destinos).

Aqui, um fluxo na rede é qualquer vetor não-negativo $y \in R^{|L|}$ : as componentes $y_{l} \geq 0$ são pensadas como fluxos elementares ao longo das diversas rotas $l$. Alternativamente, é claro, podemos trabalhar com o vetor $\boldsymbol{f}=\mathbb{I} \boldsymbol{y} \mathrm{em} R^{|K|}$, cujas componentes são os fluxos agregados induzidos nos arcos da rede. Como trabalharemos com uma rede capacitada pelo vetor $\boldsymbol{c}$, devemos limitar nossos fluxos: $\boldsymbol{f} \leq \boldsymbol{c}$ (desigualdade arco a arco: o fluxo $f_{k}$ no arco $k$ não pode ultrapassar sua capacidade $c_{k}$ ). O valor do fluxo $\boldsymbol{y}$ é $v(\boldsymbol{y})=\boldsymbol{e}^{\prime} \boldsymbol{y}=\sum_{I} f^{i}=\sum_{J} f^{j}$, onde $f^{i}=\sum_{K(i)} f_{k}$ (o fluxo total entrando na rede através da particular origem $i$ ), etc. $\mathrm{O}$ valor de um corte $C$ é $v(C)=\sum_{C} c_{k}$. Em geral temos $v(\boldsymbol{y}) \leq v(C)$ para todo fluxo $\boldsymbol{y}$ e corte $C$ na rede e, por dualidade, os valores extremos (máximo e mínimo, respectivamente) das funções $v(\boldsymbol{y}) \mathrm{e}$ $v(C)$ coincidem - o clássico teorema de Ford e Fulkerson (ver e.g., Ahuja et al., 1993 e Shrijver, 2002).

Usaremos ainda a notação $c(i), \boldsymbol{y}(j)$, etc, para representar a capacidade total $\sum_{K(i)} c_{k}$ de entrada na rede através da origem $i$, o fluxo total $\sum_{L(j)} y_{l}$ de chegada ao destino $j$, etc. Note que $\boldsymbol{y}(i) \leq c(i)$, é uma restrição natural: o fluxo total de entrada em cada origem não pode ultrapassar a capacidade total de entrada neste ponto.

\section{A Abordagem de Washburn e Wood}

Os problemas maxmin-minmax (1) podem ser formulados como um par dual em Programação Linear: 

(a) $\left\{\begin{array}{l}\min \boldsymbol{e}^{\prime} \boldsymbol{x} \\ M^{\prime} \boldsymbol{x} \geq e \\ \boldsymbol{x} \geq \mathbf{0}\end{array}\right.$
(b) $\left\{\begin{array}{l}\max \boldsymbol{e}^{\prime} \boldsymbol{z} \\ \mathbb{M} \boldsymbol{z} \leq \boldsymbol{e} \\ \boldsymbol{z} \geq \mathbf{0}\end{array}\right.$
(onde $\mathbb{M}=\mathbb{D} \mathbb{I} \mathbb{V}$ )

com soluções ótimas $\overline{\boldsymbol{x}}$ e $\bar{z}$, satisfazendo $\boldsymbol{e}^{\prime} \overline{\boldsymbol{x}}=\boldsymbol{e}^{\prime} \overline{\boldsymbol{z}}=m$, relacionadas com as estratégias ótimas $\overline{\mathbf{x}}$ e $\overline{\mathbf{y}}$ dos jogadores e com o valor do jogo $\bar{g}=g(\overline{\mathbf{x}}, \overline{\mathbf{y}})$ por $\bar{g}=1 / m, \overline{\mathbf{x}}=\overline{\boldsymbol{x}} / m$, $\overline{\mathbf{y}}=\mathbb{V} \overline{\boldsymbol{z}} / m(\overline{\boldsymbol{x}} / m$ e $\overline{\boldsymbol{z}} / m$ são as normalizações das soluções ótimas de (2)).

No problema livre, isto é, sem cotas (sem a restrição $\mathbb{R} \boldsymbol{y}=\boldsymbol{a}$ ) o par (2) tem a forma mais simples,
(a) $\left\{\begin{array}{l}\min \boldsymbol{e}^{\prime} \boldsymbol{x} \\ I^{\prime} \mathbb{D} \boldsymbol{x} \geq \boldsymbol{e} \\ \boldsymbol{x} \geq \mathbf{0}\end{array}\right.$
(b) $\left\{\begin{array}{l}\max \boldsymbol{e}^{\prime} \boldsymbol{y} \\ \mathbb{D} \mathbb{I} \boldsymbol{y} \leq \boldsymbol{e} \\ \boldsymbol{y} \geq \mathbf{0}\end{array}\right.$

o que possibilitou Washburn \& Wood (1995) reduzirem-no a um problema de fluxo max-corte min na rede $\mathfrak{R}$ capacitada pelo vetor $\boldsymbol{c}$, introduzido na Seção 2 . Algebricamente (ver Machado \& Sinotti, 2002), fazendo $\mathbb{D} \boldsymbol{x}=\boldsymbol{X}$ e observando que $\mathbb{C}$ é uma matriz não-negativa, que $\mathbb{C} \boldsymbol{e}=\boldsymbol{c}$ e $\boldsymbol{e}^{\prime} \boldsymbol{x}=\boldsymbol{c}^{\prime} \boldsymbol{X}$, obtemos o par dual:
(a) $\left\{\begin{array}{l}\min c^{\prime} \boldsymbol{X} \\ \mathbb{I}^{\prime} \boldsymbol{X} \geq \boldsymbol{e} \\ \boldsymbol{X} \geq \mathbf{0}\end{array}\right.$
(b) $\left\{\begin{array}{l}\max \boldsymbol{e}^{\prime} \boldsymbol{y} \\ \mathbb{I} \boldsymbol{y} \leq \boldsymbol{c} \\ \boldsymbol{y} \geq \mathbf{0}\end{array}\right.$

Observe que (4b) é a versão-rotas do problema de fluxo máximo, usualmente formulado em termos dos fluxos nos arcos, mas aqui, dos fluxos ao longo das rotas. Por outro lado, dado um corte qualquer $C$ na rede, tomando $\boldsymbol{X}_{k}=1$ quando $k \in C$ e $\boldsymbol{X}_{k}=0$, caso contrário, obtemos uma solução viável $\boldsymbol{X}$ de (4a), (pois toda rota passa por algum arco do corte), cujo valor-objetivo é igual a $V(C)$. A dualidade fluxo máximo-corte mínimo permite assim interpretar (4a) como o problema do corte mínimo na rede capacitada.

A superioridade da versão (4) do problema livre está no fato de dispormos de algoritmos combinatoriais eficientes (Ford e Fulkerson e variantes) para o problema do fluxo máximo, que utilizam a estrutura básica da rede diretamente, isto é, a matriz de incidência nó $\times$ arco (de ordem $|N| \times|K|$ ), dispensando assim a necessidade da especificação, a priori, de todas as possíveis rotas $l$ da rede $\mathfrak{R}$ - uma tarefa onerosa, se não impraticável, mesmo em redes de dimensões modestas (ver e.g., Sinotti, 2000). Vale observar ainda que a ordem da matriz $\mathbb{I}$, $(|K| \times|L|$, com $L$ grande), essencial à versão original (3), dificulta computacionalmente também a aplicação direta do Método Simplexo. Lastimavelmente, a vantagem computacional obtida no caso livre não se estende ao nosso problema, cotado nos extremos: as estratégias do evasor são proporcionais, isto é, devem respeitar as cotas definidas pelo vetor $\boldsymbol{a}=\left(\boldsymbol{\alpha}^{\prime}, \boldsymbol{\beta}^{\prime}\right)$. Agora o par dual (2) requer, além da especificação de todas as rotas em $\mathfrak{R}$, a determinação preliminar de todas as soluções básicas do sistema $\mathbb{R} \boldsymbol{y}=\boldsymbol{a}$ (de ordem 
$|E| \times|L|$ ), os pontos extremos $\boldsymbol{v}_{t}$ do simplexo $\mathbb{S}_{\mathrm{L}}-$ um subconjunto de distribuições sobre as rotas $l$, capaz de gerar (mediante combinações convexas) todas as distribuições proporcionais, o objeto de nosso interesse.

Ainda que algébrica e conceitualmente simples, a restrição adicional, de proporcionalidade das estratégias $\boldsymbol{y}$ do evasor, não parece ser fácil e diretamente tratável por qualquer versão, apropriadamente adaptada, do algoritmo de Ford e Fulkerson, ou outro, do mesmo tipo, na literatura, prejudicando assim a base do sucesso computacional do esquema Washburn-Wood no caso livre. Na próxima seção mostramos como o problema do evasor ainda pode ser formulado, de modo natural, como um tipo particular de problema de fluxo máximo e propomos um esquema de resolução indireto, baseado em sucessivas delimitações do fluxo, utilizando o algoritmo de Ford e Fulkerson, passo a passo, no processo iterativo.

\section{Um Esquema Iterativo}

Observe inicialmente que no problema do evasor (1b) temos, para $\boldsymbol{y} \in \mathbb{S}_{\mathrm{L}}$ fixado, que

$$
\max \left\{\boldsymbol{x}^{\prime} \mathbb{D} \mathbb{I} \boldsymbol{y}: \boldsymbol{x} \in S_{K}\right\}=\min \{\lambda: \lambda \boldsymbol{e} \geq \mathbb{D I} \boldsymbol{y}\} .
$$

De fato, o máximo é a maior das componentes do vetor $\mathbb{D} \mathbb{I} \boldsymbol{y}$ e, portanto, a menor das cotas superiores deste conjunto. Podemos portanto reescrever (1b) na forma (5) abaixo

$$
\left\{\begin{array} { l } 
{ \operatorname { m i n } \lambda } \\
{ \mathbb { D I } \boldsymbol { y } \leq \lambda \boldsymbol { e } } \\
{ \mathbb { R } \boldsymbol { y } = \boldsymbol { a } } \\
{ \boldsymbol { y } \geq \mathbf { 0 } }
\end{array} \quad \left\{\begin{array}{l}
\max \boldsymbol{e}^{\prime} \mathbf{y} \\
\mathbb{I} \mathbf{y} \leq \boldsymbol{c} \\
\mathbb{R} \mathbf{y}=\left(\boldsymbol{e}^{\prime} \mathbf{y}\right) \boldsymbol{a} \\
\mathbf{y} \geq \mathbf{0}
\end{array}\right.\right.
$$

(a restrição $\boldsymbol{e}^{\prime} \boldsymbol{y}=1$ é redundante em (5), em presença da condição $\mathbb{R} \boldsymbol{y}=\boldsymbol{a}$ ). Fazendo $\boldsymbol{y} / \lambda=\mathbf{y}$, multiplicando a desigualdade vetorial pela matriz não-negativa $\mathbb{C}$ e observando que $\boldsymbol{e}^{\prime} \mathbf{y}=1 / \lambda$, obtemos a forma final (6).

Reduzimos assim o problema do evasor à determinação do fluxo proporcional máximo na rede capacitada, isto é, o fluxo $\mathbf{y} \in R^{|\mathrm{L}|}$ de maior valor possível ( $\max \boldsymbol{e} \mathbf{y}$ ) que respeita arco a arco as diversas capacidades da rede $(\mathbb{I} \mathbf{y} \leq \boldsymbol{c})$ e os percentuais pré-estabelecidos do valor agregado $v(\mathbf{y})=\boldsymbol{e}^{\prime} \mathbf{y}, \alpha \in S_{I}$ e $\beta \in S_{J}$, nas diversas origens e nos diversos destinos (a restrição $\left.\mathbb{R}(\mathbf{y})=\left(\boldsymbol{e}^{\prime} \mathbf{y}\right) \boldsymbol{a}\right)$. Observe que ainda temos um problema de fluxo capacitado máximo; desta vez no entanto a maximização é restrita aos fluxos proporcionais: aqueles satisfazendo, para cada $i \in I$ e $j \in J$, as relações $\sum_{L(i)} \mathrm{y}_{l}=\alpha_{i}\left(\sum_{L} \mathrm{y}_{l}\right)$ e $\sum_{L(j)} \mathrm{y}_{l}=\beta_{i}\left(\sum_{L} \mathrm{y}_{l}\right)$.

Daqui em diante trabalharemos com a rede estendida $\overline{\mathfrak{R}}$, obtida a partir da original pelo método tradicional: acrescentando dois extremos artificiais, uma origem $O$ e um destino $D$ (agora únicos) e um total de $(|I|+|J|)$ arcos artificiais $O i$ e $j D$, ligando $O$ a cada uma das origens $i$ de $\mathfrak{R}$ e, cada destino $j$ a $D$. 
Se $m$ é uma cota superior qualquer para o valor do fluxo proporcional máximo $\overline{\mathbf{y}}$ em $\mathfrak{R}$, isto é, $v(\overline{\mathbf{y}}) \leq m$, capacitaremos os novos $\operatorname{arcos} O i$ e $j D$ em $\overline{\mathfrak{R}}$ por $c(O i)=\alpha_{i} m$ e $c(j D)=\beta_{j} m$.

É fácil verificar que: (1) rotas em $\Re$ (ligando $i$ a $j$ ) se identificam com rotas em $\bar{\Re}$ (ligando $O$ a $D$ e passando intermediariamente por $i$ e $j$ ), (2) um vetor $\mathbf{y} \in R^{|\mathrm{L}|}$ representa assim, tanto um fluxo em $\mathfrak{R}$, como em sua extensão $\bar{\Re}$ e (3) um fluxo que respeita as capacidades em $\bar{\Re}$ é proporcional se e somente se respeita as capacidades nos arcos artificiais da extensão e é proporcional em $\overline{\mathfrak{R}}$, no sentido de que $\mathbf{y}(O i)=\alpha_{i} v(\mathbf{y})$ e $\mathbf{y}(j D)=\beta_{J} v(\mathbf{y})$ nos $\operatorname{arcos}$ artificiais.

A busca da solução ótima $\overline{\mathbf{y}}$ do problema (6) é portanto equivalente à do fluxo proporcional máximo na extensão $\bar{\Re}$ construída a partir de uma cota superior conhecida qualquer do valor $v(\overline{\mathbf{y}})$ (em princípio, desconhecido) do fluxo proporcional máximo em $\mathfrak{R}$.

Se $\mathbf{y}$ é um fluxo proporcional qualquer temos $c(i) \geq \mathbf{y}(i)=\alpha_{i} v(\mathbf{y})$ para cada origem $i$ e algo análogo para os destinos $j$ de $\mathfrak{R}$. Assim, $m_{0}=\min _{i, j}\left\{c(i) / \alpha_{i}, c(j) / \beta_{j}\right\} \geq v(\mathbf{y})$, e temos em mãos uma majorante (a primeira delas) do valor ótimo $v(\overline{\mathbf{y}})$.

Considere inicialmente a extensão $\mathfrak{R}_{0}$ da rede original $\mathfrak{R}$, associada à esta cota (isto é, construída como indicado anteriormente, a partir do valor $m_{0}$ ) e $\mathbf{y}_{0}$ um fluxo máximo qualquer em $\bar{\Re}_{0}$. Necessariamente:

$$
v(\overline{\mathbf{y}}) \leq v\left(\mathbf{y}_{0}\right)=\sum_{I} \mathbf{y}_{0}(O i) \leq \sum_{I} c(O i)=\sum_{I} \alpha_{I} m_{0}=m_{0}
$$

e o valor $m_{1}=v\left(\mathbf{y}_{0}\right)$ é portanto uma nova majorante do valor ótimo $v(\overline{\mathbf{y}})$, tão boa quanto ou melhor do que a anterior: $m_{1} \leq m_{0}$. Caso se tenha $m_{1}=m_{0}$, segue-se da desigualdade que $\mathbf{y}_{0}(O i)=\alpha_{i} m_{0}=\alpha_{i} v\left(\mathbf{y}_{0}\right)$ para toda origem $i$, o mesmo ocorrendo nos destinos $j$ da rede $\mathfrak{R}$, e portanto $\mathbf{y}_{0}$ é um fluxo proporcional em $\overline{\mathfrak{R}}_{0}$. Por ser um fluxo máximo, $\mathbf{y}_{0}$ é necessariamente um máximo entre todos os fluxos proporcionais, isto é, $\mathbf{y}_{0}$ é um fluxo proporcional máximo e a busca de uma solução do problema (6) se encerra.

Se $m_{1}<m_{0}$ então $\mathbf{y}_{0}$ não é um fluxo proporcional ótimo (pode ser proporcional mas, em princípio temos apenas que $\left.v(\overline{\mathbf{y}}) \leq m_{1}<m_{0}\right)$ e a busca continua, construindo-se agora a extensão $\overline{\mathfrak{R}}_{1}$ da rede original $\mathfrak{R}$ utilizando a nova cota $m_{1}$ e assim por diante. O procedimento é interrompido se duas cotas sucessivas coincidem, $m_{r+1}=m_{r}$, caso em que o fluxo máximo $\mathbf{y}_{r}$ na rede estendida $\overline{\mathfrak{R}}_{r}$ é um fluxo proporcional máximo na rede $\mathfrak{R}$. Se a igualdade não ocorrer, temos uma seqüência decrescente de majorantes $m_{0}>m_{1}>\ldots>v(\overline{\mathbf{y}})$ e de fluxos $\mathbf{y}_{0}, \mathbf{y}_{1}, \ldots$ em $\mathfrak{R}$, com $m_{r+1}=v\left(\mathbf{y}_{r}\right)$. Como veremos adiante, é possível mostrar que: (1) o valor limite $m^{*}=\lim _{\infty} m_{r}$ coincide com $v(\overline{\mathbf{y}})$ e (2) todo ponto limite $\mathbf{y}^{*}$ da sequiência $\mathbf{y}_{r} \in R^{|\mathrm{L}|}$, isto é, limite de qualquer de suas subseqüências, é um fluxo proporcional máximo na rede $\mathfrak{R}$, isto é, uma solução do problema (6). 


\section{A Convergência do Algoritmo}

Na seção anterior introduzimos o esquema: majorante $m_{r} \rightarrow$ extensão $\tilde{\mathfrak{R}}_{r}\left(\operatorname{com} c_{r}(i)=\alpha_{i} m_{r}\right.$, $\left.c_{r}(j)=\beta_{j} m_{r}\right) \rightarrow \mathbf{y}_{r}$ fluxo máximo $\rightarrow \boldsymbol{v}\left(\mathbf{y}_{r}\right)=m_{r+1}$, nova majorante. Admitiremos aqui que a sequiência $m_{r}$ é infinita (caso contrário, temos uma solução do problema original (6): a primeira possibilidade mencionada anteriormente), isto é, $m_{0}>m_{1}>m_{2}>\ldots$, decrescendo para um valor limite $m^{*} \geq v(\overline{\mathbf{y}})$, onde $\overline{\mathbf{y}}$ é um fluxo proporcional máximo (a ser determinado). Mostraremos a seguir, de forma construtiva, que $m^{*}=v(\overline{\mathbf{y}})$, isto é que $m^{*}$ é o valor do fluxo proporcional ótimo.

Observe inicialmente que, nos $\operatorname{arcos} k, 0 \leq \mathbf{y}_{r}(k) \leq c_{k}$ e, nos arcos artificiais iniciais, $0 \leq \mathbf{y}_{r}(i)=\mathbf{y}_{r}(O i) \leq \alpha_{i} m_{r}$, por construção, o mesmo ocorrendo com os terminais $j D$ em $\mathfrak{R}_{r}$ (mesma observação, por default, daqui em diante).

A seqüência de extensões $\tilde{\mathfrak{R}}_{r}$ associadas às majorantes $m_{r} \geq v(\overline{\mathbf{y}})$, converge para a extensão $\tilde{\mathfrak{R}}^{*}$ associada à majorante $m^{*}$. De fato, nos arcos $k$, a capacidade $c_{k}$ é a mesma para todas as extensões, e nos arcos artificiais $O i$, temos $c_{r}(i)=\alpha_{i} m_{r}$ que converge (decrescentemente) para $\alpha_{i} m^{*}$, que é a capacidade $c^{*}(i)$ em $\tilde{\mathfrak{R}}^{*}$.

Note ainda que a sequiência dos fluxos $\mathbf{y}_{r} \in R^{|L|}$ é limitada a um politopo $P$ definido pelas desigualdades $\mathbf{y}_{r} \geq \mathbf{0}$ e $\boldsymbol{e}^{\prime} \mathbf{y}_{r}=v\left(\mathbf{y}_{r}\right)=m_{r+1} \in\left[m^{*}, m_{0}\right]$, e que, portanto, alguma subseqüência, que denotaremos por $\mathbf{y}_{s}$, da seqüência original $\mathbf{y}_{r}$ converge (devido à compacidade de $P$ ). Seja $\mathbf{y}^{*}$ o limite de uma seqüência deste tipo, isto é, $\lim _{\infty} \mathbf{y}_{s}=\mathbf{y}^{*}$. Temos $\lim _{\infty} \mathbf{y}_{s}(k)=\mathbf{y}^{*}(k)$ para os $\operatorname{arcos} k$ da rede original e $\lim _{\infty} \mathbf{y}_{s}(i)=\mathbf{y}^{*}(i)$ para as origens $i$. De fato, como $\mathbf{y}_{s}(k)=\sum_{l(k)}\left(\mathbf{y}_{s}\right)_{l}$ e $\mathbf{y}_{s}(i)=\sum_{L(i)}\left(\mathbf{y}_{s}\right)_{l}$ (soma de componentes do vetor $\mathbf{y}_{s}$ ), basta passar a primeira expressão ao limite para obter a segunda. Por sua vez, das restrições de capacidade nos $\operatorname{arcos} k \in K$ e nas origens $i \in I$, temos, no limite: $0 \leq \mathbf{y}_{r}(k) \leq c_{k}$ e $\mathbf{0} \leq \mathbf{y}^{*}(i) \leq \alpha_{i} m^{*}$, isto é, $\mathbf{y}^{*}$ é um fluxo na rede capacitada $\tilde{\mathfrak{R}}^{*}$.

Vale também a igualdade $v\left(\mathbf{y}^{*}\right)=m^{*}$, isto é, o valor do fluxo limite é o limite dos valores dos fluxos $\mathbf{y}_{s}$ : basta observar que $v\left(\mathbf{y}_{s}\right)=\sum_{L}\left(\mathbf{y}_{s}\right)_{l}$ (o mesmo valendo para $\mathbf{y}^{*}$ ) e passar a expressão ao limite, como antes.

$\mathrm{O}$ vetor $\mathbf{y}^{*}$ é um fluxo proporcional em $\mathfrak{R}^{*}:$ da desigualdade $m^{*}=v\left(\mathbf{y}^{*}\right)=\sum_{I} \mathbf{y}^{*}(i) \leq$ $\sum_{I} \alpha_{i} m^{*}=m^{*}$ segue a igualdade das parcelas, $\mathbf{y}^{*}(i)=\alpha_{i} m^{*}$. Além disso, $\mathbf{y}^{*}$ é um fluxo máximo na rede $\tilde{\mathfrak{R}}^{*}$ : os arcos artificiais $O i$ formam um corte $C^{*}$ com valor $v\left(C^{*}\right)=$ $\sum_{I} C^{*}(O i)=\sum_{I} \alpha_{i} m^{*}=m^{*}=v\left(\mathbf{y}^{*}\right), \operatorname{logo}$, pelo Teorema de Dualidade de Ford e Fulkerson, o fluxo $\mathbf{y}^{*}$ é máximo. 
Finalmente, como $\mathbf{y}^{*}$ é proporcional e é também um fluxo máximo na rede estendida $\tilde{\mathfrak{R}}^{*}$ associada à cota $m^{*}=v(\overline{\mathbf{y}}), \mathbf{y}^{*}$ é um fluxo proporcional máximo na rede original $\mathfrak{R}$, com valor $m^{*}$.

Fica assim provado que, se o algoritmo descrito não fornecer uma solução ótima $\overline{\mathbf{y}}$ em um número finito de passos, qualquer ponto limite da sequiência dos fluxos $\mathbf{y}_{r}$ obtidos iterativamente no processo, é uma solução e que a seqüência decrescente de majorantes $m_{r}$, gerada pelo algoritmo, converge para o valor ótimo, isto é, para o maior dos valores possíveis entre os fluxos proporcionais na rede $\mathfrak{R}$.

Lembramos aqui que as soluções $\mathbf{y}$ do problema de fluxo proporcional máximo e $\boldsymbol{y}$ do problema do evasor estão relacionadas por $\boldsymbol{y}=\mathbf{y} / \boldsymbol{\lambda}$ onde $\boldsymbol{e}^{\prime} \mathbf{y}=1 / \boldsymbol{\lambda}$ (a mesma relação do esquema Washburn-Wood para a resolução do problema livre, isto é, sem cotas estabelecidas nas origens e destinos). Assim, se $\overline{\mathbf{y}}$ é um fluxo proporcional máximo na rede $\mathfrak{R}$ (capacitada por $c_{k}=1 / p_{k}$, arco a arco) com valor $v(\overline{\mathbf{y}})=\boldsymbol{e}^{\prime} \overline{\mathbf{y}}$, então o valor do jogo de confronto (com probabilidades $p_{k}$ de detecção nos arcos) é $\bar{g}=\bar{\lambda}=1 / v(\overline{\mathbf{y}})$ e a estratégia ótima do evasor é $\overline{\boldsymbol{y}}=\bar{\lambda} \overline{\mathbf{y}}=\overline{\mathbf{y}} / v(\overline{\mathbf{y}})$.

\section{Um Exemplo de Implementação}

Ilustraremos agora a implementação do algoritmo da Seção 5 a uma rede simples $\mathfrak{R}$, representada abaixo, com 12 nós, mais origem e destino artificiais, aqui denotados por $\mathbf{O}$ e $\mathbf{M}, 17$ arcos, mais 4 artificiais, e um total de 23 rotas ligando $\mathbf{O}$ e $\mathbf{M}$.

Admitiremos aqui, que o problema de confronto evasor $\times$ detector já está na forma final (6), da Seção 4, isto é, de um problema de fluxo máximo proporcional. Assim, as capacidades arbitradas nos diversos arcos da Figura 1, e.g. $c(\mathbf{A E})=18$ e $c(\mathbf{F G})=15$, correspondem às probabilidades de detecção no problema original, $p(\mathbf{A E})=1 / 18=5,6 \%$ e $p(\mathbf{F G})=1 / 15=$ $6,7 \%$, percentuais aproximados, indicados entre parênteses. Arbitramos ainda as cotas $\alpha=(0,80,0,20)$ e $\beta=(0,75,0,25)$ para as origens $A$ e $B$ e os destinos $K$ e $L$, respectivamente.

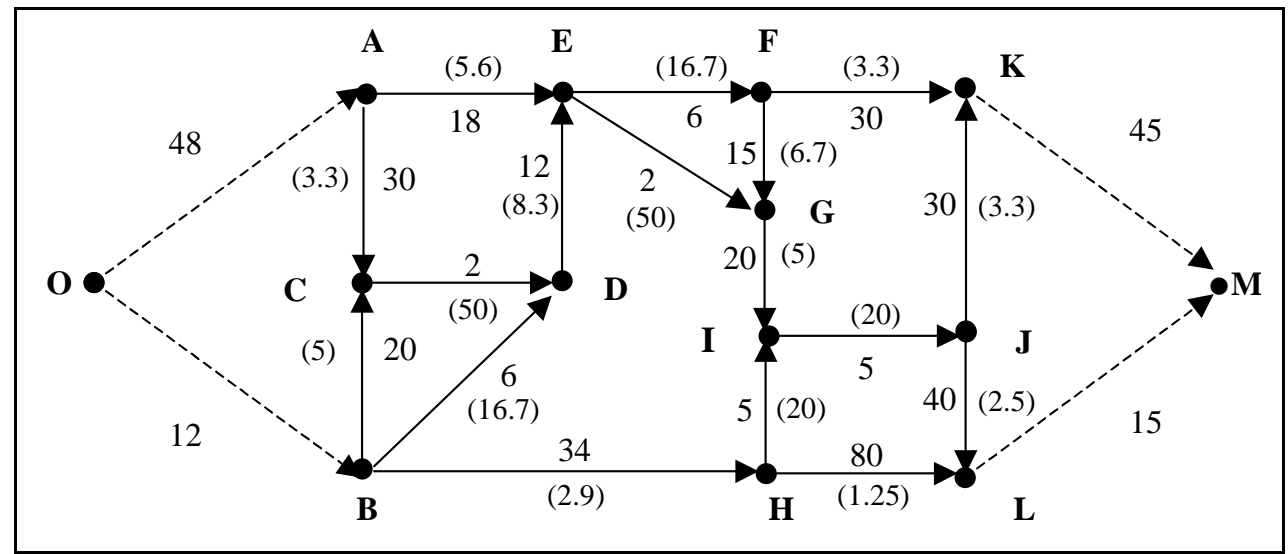

Figura 1 - A rede $\mathfrak{R}$ e a extensão inicial $\tilde{\mathfrak{R}}_{0}$. 
Listados lexicograficamente, temos aqui os seguintes conjuntos (vide Seção 2): $\mathbf{N}=\{\mathbf{A}$, $\mathbf{B}, \ldots, \mathbf{L}\}$ de 12 nós, $K=\{\mathbf{A C}, \mathbf{A E}, \mathbf{B C}, \ldots, \mathbf{J L}\}$ de $17 \operatorname{arcos}, \mathbf{E}=\{\mathbf{A}, \mathbf{B}, \mathbf{K}, \mathbf{L}\}$ de 4 extremos e L, das 23 rotas, $l_{1}=$ ACDEFGIJK, $l_{2}=$ ACDEFGIJL,$l_{3}=$ ACDEFK $, \ldots, l_{23}=\mathbf{B H L}$. Correspondentemente, temos as matrizes $\mathbb{C}=\operatorname{diag}(30,18,20, \ldots, 40)$ de capacidades e sua inversa $\mathbb{D}=\operatorname{diag}(1 / 30,1 / 18,1 / 20, \ldots, 1 / 40)$ de probabilidades de detecção nos arcos (ordem 17), de incidência arco $\times$ rota $\mathbb{I}$, com $\mathbb{I}_{1,1}=1$ (o $1^{\circ}$ arco está na $1^{\text {a }}$ rota), $\mathbb{I}_{17,23}=0$ (o arco $\mathbf{J L}$ não pertence à rota $\mathbf{B L H})$, etc., e extremo $\times$ rota $\mathbb{R}, \operatorname{com} \mathbb{R}_{1,1}=1\left(\boldsymbol{A} \in l_{1}\right)$, $\mathbb{R}_{3,23}=0\left(\boldsymbol{K} \notin l_{23}\right)$, etc. Temos ainda os símplices $S_{L}$ das estratégias (livres) do evasor, $\boldsymbol{x}=\left(x_{1}, \ldots, x_{23}\right)$ com $x_{l} \geq 0, \sum x_{l}=1, S_{K}$ das estratégias do detector, $\boldsymbol{y}=\left(y_{1}, \ldots, y_{17}\right)$, onde $y_{k} \geq 0, \sum y_{k}=1$ e $\mathbb{S}_{L}$, das estratégias proporcionais do evasor, isto é, soluções do sistema $\boldsymbol{y} \in S^{|L|}, \mathbb{R} \boldsymbol{y}=\boldsymbol{a}$ (sendo $\boldsymbol{a}=(0,80,0,20,0,75,0,25)$ ), que, pode-se mostrar, possui mais de 300 soluções básicas (isto é, $T \geq 300$ ), entre outras, $\bar{y}$ e $\tilde{y}$, onde $\bar{y}_{l}=0,75,0,05$ ou 0,20 para as rotas $l_{8}=\mathbf{A E F K}, l_{10}=\mathbf{A E G I J L}$ ou $l_{23}=\mathbf{B H L}$, respectivamente, e $\bar{y}_{l}=0$, caso contrário, e $\tilde{y}_{l}=0,55,0,25$ ou 0,20 , para as rotas $l_{1}=$ ACDEFGIJK, $l_{10}=$ AEGIJL ou $l_{21}=$ BHIJK, respectivamente e $\tilde{y}_{l}=0$, caso contrário.

Os arcos artificiais, que receberiam normalmente as capacidades agregadas: $c(\mathbf{O A})=30+18$ $=48, c(\mathbf{O B})=20+6+34=60, c(\mathbf{K M})=30+30=60$ e $c(\mathbf{L M})=80+40=120$, aqui receberam, no $1^{\circ}$ passo do algoritmo, percentuais apropriados da cota superior inicial $m_{0}=\min \{48 / 0,80,60 / 0,20,60 / 0,75,120 / 0,25\}=\min \{60,300,80,480\}=60$. Assim, $c(\mathbf{O A})=0,80 \times m_{0}=48, c(\mathbf{O B})=0,20 \times m_{0}=12, c(\mathbf{K M})=0,75 \times m_{0}=45$ e $c(\mathbf{L M})=0,25 \times$ $m_{0}=15$, conforme indicado na Figura 1 e na $1^{\text {a }}$ coluna da Tabela 2, mais adiante. Esta é a nossa extensão inicial $\tilde{R}_{0}$ da rede $\mathfrak{R}$ original (sem origem e destino artificiais). Na $1^{\text {a }}$ coluna da Tabela 1, a seguir, indicamos, arco a arco, o fluxo máximo $\overline{\mathbf{y}}_{0}$ (ou melhor, sua versão-arcos) em $\tilde{\mathfrak{R}}_{0}$, com valor $m_{1}=v\left(\overline{\mathbf{y}}_{0}\right)=20$, o qual aparece na última linha. Isto encerra o $1^{\circ}$ passo na implantação do algoritmo.

Observe que $\mathbf{O B}, \mathbf{E F}$ e $\mathbf{E G}$ (com asteriscos na tabela) formam um corte de $\mathfrak{R}_{0}$ com valor $c(\mathbf{O B})+c(\mathbf{E F})+c(\mathbf{E G})=12+6+2=20$, coincidente com o valor do fluxo $\mathbf{y}_{0}$, o que comprova a maximilidade em $\mathfrak{R}_{0}$. Temos assim uma nova cota superior $m_{1}=20$ da solução proporcional ótima, e com ela iniciamos o $2^{\circ}$ passo do algoritmo. Agora, $c(\mathbf{A O})=0,8 m_{1}=16$, $c(\mathrm{OB})=0,2 m_{1}=4$, etc, valores figurando na $2^{\mathrm{a}}$ coluna da Tabela 2. Nesta nova extensão $\overline{\mathfrak{R}}_{1}$ de $\mathfrak{R}$, resolvendo o problema do fluxo máximo, obtemos uma solução (indicada na $2^{\mathrm{a}}$ coluna da Tabela 1) com valor 12 que serve agora como nova cota superior $m_{2}$, utilizada para iniciar a $3^{\mathrm{a}}$ iteração, e assim por diante. $O$ processo foi levado até a $5^{\mathrm{a}}$ iteração e os resultados indicados nas Tabelas 1 (fluxos máximos) e $\mathbf{2}$ (cotas sucessivas $m$ e as capacidades dos arcos artificiais nas diversas extensões $\mathfrak{R}_{r}$ ). 
Tabela 1 - Fluxos máximos

\begin{tabular}{ccccccc}
\hline & \multicolumn{5}{c}{ Iteração $r$} & \multirow{2}{*}{$\boldsymbol{f}^{*}$} \\
\cline { 2 - 5 } & $\mathbf{1}$ & $\mathbf{2}$ & $\mathbf{3}$ & $\mathbf{4}$ & $\mathbf{5}$ & \\
\hline $\mathbf{A O}$ & 8 & 8 & 8 & 8 & 8 & 8 \\
$\mathbf{O B} *$ & 12 & 4 & 2,4 & 2,08 & 2,016 & 2 \\
$\mathbf{A E}$ & 8 & 8 & 8 & 8 & 8 & 8 \\
$\mathbf{B H}$ & 12 & 4 & 2,4 & 2,08 & 2,016 & 2 \\
$\mathbf{E F} *$ & 6 & 6 & 6 & 6 & 6 & 6 \\
$\mathbf{E G} *$ & 2 & 2 & 2 & 2 & 2 & 2 \\
$\mathbf{F K}$ & 6 & 6 & 6 & 6 & 6 & 6 \\
$\mathbf{G I}$ & 2 & 2 & 2 & 2 & 2 & 2 \\
$\mathbf{H I}$ & 0 & 1 & 1,4 & 1,48 & 1,496 & 1,5 \\
$\mathbf{H L}$ & 12 & 3 & 1 & 0,60 & 0,520 & 0,5 \\
$\mathbf{I J}$ & 2 & 3 & 3,4 & 3,48 & 3,496 & 3,5 \\
$\mathbf{J K}$ & 0 & 1 & 1,4 & 1,48 & 1,496 & 1,5 \\
$\mathbf{J L}$ & 2 & 2 & 2 & 2 & 2 & 2 \\
$\mathbf{K M}$ & 6 & 7 & 7,4 & 7,48 & 7,496 & 7,5 \\
$\mathbf{L M}$ & 14 & 5 & 3 & 2,60 & 2,520 & 2,5 \\
\hline$v\left(\mathbf{y}_{r}\right)=m_{r+1}$ & 20 & 12 & 10,4 & 10,08 & 10,016 & 10 \\
\hline & & & & & &
\end{tabular}

Na última coluna da Tabela 1, aparece uma solução ótima $\overline{\mathbf{y}}$, isto é, um fluxo proporcional máximo em $\mathfrak{R}$ (descrito em termos de arcos). De fato, como toda rota originando em $\mathbf{A}$ passa por um dos arcos EF e EG, com capacidade agregada $6+2=8$, qualquer fluxo $\mathbf{y}$ na rede tem no máximo este valor associado ao nó $\mathbf{A}(\mathbf{y}(\mathbf{A}) \leq 8$, na notação da Seção 2$)$. Se $\mathbf{y}$ for proporcional, seu valor $v(\mathbf{y})$ é no máximo $8 / 0,80=10$, o valor do fluxo (proporcional) em questão. No nosso exemplo, bastante simples, é possível mostrar a relação $m_{r+1}=8+0,2 m_{r}$, entre as cotas sucessivas $m_{r}$ e então, passando ao limite quando $r \rightarrow \infty$, temos que $m^{*}=8+0,2 m^{*}$, isto é, $m^{*}=10$, uma confirmação alternativa da otimalidade. Vale a pena observar que aqui não podemos usar a dualidade fluxo máximo-corte mínimo (o corte de menor valor na rede $\mathfrak{R}$ tem valor 20 , igual ao do fluxo máximo $\mathbf{y}_{0}$ ) pois a maximalidade aqui é restrita aos fluxos proporcionais e não livre.

Na última coluna da Tabela 2, indicamos os valores iniciais $m_{5}$ e das capacidades nos arcos artificiais da rede $\overline{\mathfrak{R}}_{6}$ que seriam utilizados na $6^{\mathrm{a}}$ iteração. Sua proximidade, respectivamente, dos valores $m^{*}, 0,80 m^{*}, 0,20 m^{*}, 0,75 m^{*}$ e $0,25 m^{*}$ destacam quão próximos da proporcionalidade desejada estamos. Por sua vez, os fluxos sucessivos na Tabela 1 indicam o grau de aproximação da solução ótima, arco a arco. 
Tabela 2 - Capacidades nos Arcos Artificiais.

\begin{tabular}{c|ccccccc}
\hline & \multicolumn{7}{c}{ Iteração } \\
\cline { 2 - 8 } Cotas & $\mathbf{1}$ & $\mathbf{2}$ & $\mathbf{3}$ & $\mathbf{4}$ & $\mathbf{5}$ & $\mathbf{6}$ \\
\cline { 2 - 8 } \multicolumn{2}{c}{ Sucessivas } & 60 & 20 & 12 & 10,4 & 10,08 & 10,016 \\
\hline \multirow{4}{*}{ Arcos } & OA & 48 & 16 & 9,6 & 8,32 & 8,064 & 8,0128 \\
& OB & 12 & 4 & 2,4 & 2,08 & 2,016 & 2,0032 \\
& KM & 45 & 15 & 9 & 7,8 & 7,56 & 7,512 \\
& LM & 15 & 5 & 3 & 2,6 & 2,52 & 2,504 \\
\hline
\end{tabular}

Observe que a Tabela 1 especifica, na realidade, os sucessivos fluxos máximos em termos dos arcos da rede (e não das rotas, o que nos interessa na busca da estratégia ótima de evasão).

A relação $\boldsymbol{f}=\mathbb{I} \mathbf{y}$ (Seção 2) tem inversa multívoca; é possível, ainda que em princípio computacionalmente oneroso, recuperar uma das inversas $\mathbf{y} \in R^{|L|}$ (versão-rotas do fluxo) associadas a um dado $\boldsymbol{f} \in R^{|K|}$ (versão-arcos). É importante notar no entanto, que o algoritmo de Ford e Fulkerson (e variantes), utilizado no cálculo de $\boldsymbol{f}$, baseia-se na obtenção, passo a passo, dos chamados percursos de aumento (ver e.g. Boaventura Netto, 1996) e portanto fornece automaticamente um conjunto de fluxos elementares, isto é, de fluxos individuais $y_{l}$ ao longo de diferentes rotas $l \in L$ - um vetor $\mathbf{y} \in R^{|L|}$, com fluxos nos extremos satisfazendo as cotas estipuladas $\left(\boldsymbol{y}(i)=f^{i}=\alpha_{i} v(\mathbf{y})\right.$; o mesmo para os destinos $j$ ) isto é, proporcionais, de valor agregado $v(\mathbf{y})=\sum_{I} f^{i}=\sum_{J} f^{j}$ máximo, portanto, uma solução da versão (6) do problema do evasor, da qual se obtém diretamente a solução do problema original (vide Seção 2).

No nosso exemplo numérico, o algoritmo de Ford e Fulkerson aplicado à rede $\mathfrak{R}_{5}$ fornece os seguintes fluxos elementares $\mathbf{y}_{l}=6,2,1,496$ e 0,520 para as rotas $l_{8}=\mathbf{A E F K}$, $l_{10}=$ AEGIJL, $l_{22}=$ BHIJL e $l_{23}=\mathbf{B H L}$, respectivamente $\left(\mathbf{y}_{l}=0\right.$ para as outras rotas $)$ com valor total $v(\mathbf{y})=10,016$, já indicado na Tabela 1. A solução $\boldsymbol{y}$ do problema do evasor é obtida por "normalização" do vetor $\mathbf{y}: \boldsymbol{y}=\mathbf{y} /(v(\mathbf{y}))$ isto é, sua estratégia ótima no confronto é utilizar (apenas) as rotas indicadas acima, com frequêências $\boldsymbol{y}_{8}=6 / 10,016=0,5990$, $\boldsymbol{y}_{10}=0,1997, \boldsymbol{y}_{22}=0,1494$ e $\boldsymbol{y}_{23}=0,0519$, respectivamente $(60,20,15$ e 5 pontos percentuais). $\mathrm{O}$ valor do jogo - a probabilidade média de detecção (admitida a racionalidade dos jogadores) é $1 / v(\mathbf{y})=0,0998$ ou seja, $10 \%$.

\section{Testes Numéricos}

Descrevemos a seguir experimentos computacionais com o algoritmo proposto, na resolução de um problema de confronto com cotas, numa rede $\mathfrak{R}$ de porte médio não trivial, mas estruturalmente simples, topológica e probabilisticamente, utilizada na literatura recente para testes em confrontos não-cotados (Sinotti, $2000 \mathrm{e}$, em dimensões inferiores, Cormican et al., 1988). Admitiremos sempre que os problemas já tenham sido reformulados em termos de fluxos proporcionais máximos - em essência, que geramos diretamente em cada simulação as capacidades $c_{k} \geq 1$ nos diversos arcos ( $1 / c_{k}$ sendo portanto as probabilidades de deteç̧ão $p_{k}$ nos problemas de confronto originais). 
A estrutura básica utilizada foi de uma malha retangular plana $\mathfrak{R}, 10 \times 11$ (linhas e colunas), com 3 origens (posições 1, 8 e 11 da coluna 1) e 4 destinos (coluna 11, posições 1, 4, 8 e 11), gerando um total de 112 nós (dois artificiais: $O$ e $D$ ) e 206 arcos orientados verticalmente ao longo das colunas de modo aleatório, com $p(\uparrow)=p(\downarrow)=0,50$ (sentidos equiprováveis, exceto, é claro, no caso de arcos iniciais e terminais) e horizontalmente, no sentido do nó artificial terminal $\mathbf{D}$.

Os parâmetros utilizados nas 16 simulações realizadas foram $\alpha=(0,50,0,35,0,15)$ para a distribuição de freqüências nas origens $l, \beta=(0,40,0,30,0,20,0,10)$ nos destinos (as freqüências de uso dos extremos pelo evasor, no confronto original), e, essencialmente, os valores $8,10,15$, e 50, para as capacidades dos arcos da rede, com exceção dos arcos iniciais (originando em $I$ ) e terminais (atingindo $J$ ), a maioria dos quais recebeu capacidades mais altas $(24,40,50,75$ e 120). A tabela abaixo ilustra os resultados obtidos.

Tabela 3 - Tempo, $n^{\circ}$ de iterações e fluxo máximo de cada simulação

\begin{tabular}{cccc}
\hline $\mathbf{N}^{\mathbf{o}}$ & Tempo $(\mathbf{s e g})$ & $\mathbf{N}^{\mathbf{o}}$ de iterações & Fluxo máximo \\
\hline 1 & 1,47 & 16 & 70 \\
2 & 1,71 & 15 & 50 \\
3 & 1,05 & 11 & 80 \\
4 & 1,59 & 16 & 60 \\
5 & 1,22 & 17 & 60 \\
6 & 1,58 & 17 & 66 \\
7 & 1,55 & 16 & 56 \\
8 & 1,87 & 18 & 52 \\
9 & 1,03 & 11 & 65,7145 \\
10 & 1,80 & 16 & 72 \\
11 & 1,65 & 15 & 62 \\
12 & 1,23 & 17 & 56 \\
13 & 1,60 & 16 & 66 \\
14 & 1,79 & 17 & 52 \\
15 & 1,80 & 19 & 60 \\
Média & $\mathbf{1 , 5 3}$ & $\mathbf{1 5 , 8 0}$ & - \\
Desvio Padrão & $\mathbf{0 , 2 7}$ & $\mathbf{2 , 2 1}$ & - \\
\hline
\end{tabular}

Nota: a $16^{\text {a }}$ simulação está relatada em detalhe no texto.

A atribuição de valores, $k \rightarrow c_{k}=8,10,15$ ou 50, isto é, a geração do vetor $c$ foi feita aleatoriamente, arco a arco, simulação a simulação, segundo a distribuição $(0,15,0,35$, $0,35,0,15)$. A escolha dos valores $c_{k}$ e das freqüências utilizadas em sua geração, ainda que arbitrárias, procurou sugerir situações plausíveis de chances mais altas, baixas ou médias de detecção ao longo da rede, neste caso, percentuais aproximados de 12,5, 10, 7 e 2 , respectivamente (lembramos que $p_{k}=1 / c_{k}$ ). Os valores mais altos atribuídos aos extremos visaram afastar casos computacionalmente simples (permitindo, em princípio, uma maior diversidade de fluxos através da rede). 
O arco de retorno, de $D$ para $O$, e os artificiais $O i$ e $j D(i \in I, j \in D)$ são inicialmente capacitados com $c=9.999 .999$ (um número "suficientemente" alto). Lembramos que o algoritmo modifica passo a passo as capacidades nos arcos artificiais, isto é, a extensão $\overline{\mathfrak{R}}$ da rede original $\mathfrak{R}$.

A resolução numérica do problema utilizou o módulo OR (Operations Research) do software SAS System, versão 8.00, através da procedure NETFLOW e foi implementada num PC Intel Pentium 4 (1.400 Mhz, 128.0 MB RAM). A geração pseudo-randômica das orientações verticais e das capacidades nos arcos interiores de $\mathfrak{R}$ foi feita automaticamente pelo programa. Uma sub-rotina auxiliar foi implementada no próprio módulo OR do SAS, como macro destinada a atualizar iterativamente as capacidades nos arcos artificiais. Estabelecemos 19 com limite para o número de iterações em cada simulação para poder observar o comportamento das sucessivas soluções aproximadas: valor do fluxo máximo, correções de proporcionalidade, rotas, cortes, etc, passo a passo. Fixamos ainda o valor $\varepsilon=0,00001$ como nível da precisão desejada que, uma vez atingida (fluxos sucessivos com valores $m_{r}$ e $\left.m_{r+1} \operatorname{com}\left(m_{r}-m_{r+1}\right) / m_{r} \leq \varepsilon\right)$, interrompia a execução da simulação em curso.

Como ilustração, descreveremos a seguir o comportamento computacional e os resultados observados na $16^{\mathrm{a}}$ simulação:

(1) A rede resultante envolve cerca de 58.000 rotas entre $\boldsymbol{O}$ e $\boldsymbol{M}$ e, portanto, cerca de 12 milhões de combinações $(l, k)$ possíveis, isto é, pares de estratégias puras do evasor (a escolha da rota de fuga $l$ ) e do detector (o local de patrulha). Quanto às mistas $(\boldsymbol{x}, \boldsymbol{y})$, é claro, apenas um subconjunto "reduzido", $S_{K} \times S_{L}$, do produto $S_{K} \times S_{L}$, respeita as cotas estabelecidas $\boldsymbol{a}=\left(\alpha^{\prime}, \beta^{\prime}\right)$.

(2) Em linhas gerais, para um valor ótimo de $v(\overline{\mathbf{y}})=68$, os erros relativos observados, $\varepsilon_{r}=\left(v\left(\mathbf{y}_{r}\right)-v(\overline{\mathbf{y}})\right) / v(\overline{\mathbf{y}})$ foram inferiores a $1 \%$ já na $5^{\mathrm{a}}$ iteração e a $0,01 \%$ na $12^{\mathrm{a}}$ iteração, atingido o nível de precisão estabelecido, $\varepsilon=0,001 \%$, apenas no $15^{\circ}$ passo.

(3) Comportamento semelhante ocorreu com os fluxos individuais nos extremos, o principal indicador do grau de aproximação à proporcionalidade desejada.

(4) Do total de 206 arcos da rede, apenas 56\% são ativados (têm fluxos não-nulos) na $1^{\text {a }}$ iteração, dos quais apenas $80 \%$ se mantêm ativos da $6^{\mathrm{a}}$ iteração em diante, o mesmo se aplicando portanto às rotas associadas, para uso ótimo do evasor no jogo original. Os arcos mantidos após a $5^{\mathrm{a}}$ iteração foram observados se agrupar em 12 classes distintas e os fluxos em cada uma delas são idênticos, arco a arco, indicando assim o total máximo de 12 trajetórias após a síntese $\boldsymbol{f} \in R^{|K|} \rightarrow \boldsymbol{y} \in R^{|L|}$ (vide Seção 2). Lembramos aqui que a representação $\boldsymbol{f}$ é suficiente para descrever a estratégia ótima do evasor que (alternativamente à recuperação das rotas a partir dos fluxos nos arcos) pode ser explicitada em termos estocásticos, nó a nó, ao longo da rede (ver e.g., Sinotti, 2000).

(5) O tempo total de CPU gasto nesta simulação foi comparativamente alto, de 4,2 seg., devido à listagem, neste caso, de resultados intermediários, arco a arco, iteração por iteração, por parte do SAS. 


\section{Conclusões}

O algoritmo proposto, baseado na delimitação forçada, sucessiva, de fluxos máximos em extensões apropriadas da rede $\mathfrak{R}$, resolve o problema do evasor em redes com múltiplas origens e destinos cotados. O resultado teórico é exato no sentido de que (1) se o procedimento para, temos a solução ótima desejada, (2) caso contrário, prossegue monotonicamente, isto é, com melhoramentos sucessivos na direção do ótimo.

Conforme ressaltado, a técnica contorna o sério problema da determinação prévia do conjunto $L$ de todas as rotas na rede e do cálculo dos pontos extremos do politopo $\mathbb{S}_{L}$, ambas tarefas problemáticas do ponto de vista computacional, dadas as cardinalidades e dimensões envolvidas, mesmo em problemas de porte reduzido, no que se refere a nós e arcos - veja e.g., a discussão (após a Figura 1) do exemplo numérico de baixa dimensão resolvido na Seção 6. Vale ressaltar que, embora os resultados da Seção 5 permitam reformular (6) em termos dos arcos da rede, isto é, a versão-arcos $\boldsymbol{f}$ do fluxo y (vide Seção 2), envolvendo a matriz de incidência nó $\times$ arco, e não a matriz arco $\times$ rota II (mais as condições lineares sobre os extremos da rede), possibilitando portanto a resolução direta, pelo Método Simplex digamos (ver e.g., Gass, 1996), restaria ainda o problema, computacionalmente árduo da síntese em termos de rotas: obter, a partir do solução ótima $\overline{\boldsymbol{f}}$, o fluxo ótimo $\overline{\mathbf{y}}$, do qual se extrairia, por normalização, a estratégia ótima de evasão $\overline{\boldsymbol{y}}$.

Quanto ao desempenho numérico do esquema iterativo proposto na Seção 4, baseado no algoritmo de Ford e Fulkerson, acreditamos que os resultados observados nas simulações efetuadas com a rede de porte médio escolhida, apesar da limitação e do caráter preliminar do experimento, apontam na direção de comprovar a eficiência computacional esperada do algoritmo.

Linhas naturais para investigação no problema evasor $\times$ detector aqui relatado (algumas delas já ativadas) incluem, entre outras:

(1) Validação empírica, estatística, mediante simulações mais extensas e problemas de ainda maior porte, da qualidade do método.

(2) A implementação do algoritmo em estruturas topológicas distintas, como em Steinrauf (1991) (diagrama simplificado de bacia fluvial na fronteira Brasil-Bolívia, grande escoadouro de insumos, isto é, precursores químicos, e produtos da coca) e Lima \& Muniz (2002) (uma estrutura radial, sugerindo níveis sucessivos de distribuição no tráfico urbano, com cinco níveis, 64 nós, mais de 150 arcos e 2.200 rotas).

(3) A busca de limites teóricos nas taxas de aproximação, visando formular regras de parada, avaliação de erros e questões de complexidade computacional.

(4) A possível recuperação da solução (ainda que aproximada) do problema primal, do detector, diretamente a partir da resolução do problema do fluxo proporcional máximo, associado ao evasor.

(5) A questão do uso de funções alternativas de avaliação (pay-off) no confronto, em particular a referente ao arrependimento máximo dos jogadores, ver Machado \& Sinotti (2002) (no presente trabalho utilizamos a probabilidade média de detecção, $g(\boldsymbol{x}, \boldsymbol{y})$ como valor do jogo). 
(6) Variantes do problema básico de confronto, como em Hohzaki \& Iida (1996), que discutem estratégias de busca arco a arco, ao longo de uma rota de percurso pré-fixada pelo detector (uma rota de detecção), levando em consideração a troca custo $\times$ benefício (agregados) das eventuais inspeções locais, a possibilidade de utilizar recursos distintos na interceptação do evasor, digamos múltiplas unidades na inspeção simultânea em diferentes arcos da rede, como em Washburn \& Wood (1995); mais geralmente, de recursos escassos diversos, a serem utilizados de modo independente, ao longo de $\Re$, como sugerido por Israeli (1999) (a probabilidade de detecção e.g., poderia depender da combinação local dos recursos); questões de vulnerabilidade em redes como em Mehdi (1994), Grötschel et al. (1998) e Bell (2003); modelos envolvendo incerteza, informação parcial, dinâmica temporal, estocacidade, etc.

\section{Agradecimentos}

Aos revisores, por muitas e valiosas críticas e sugestões e a Vinicius Rabbi Vivaldi, pelo eventual apoio técnico.

\section{Referências Bibliográficas}

(1) Ahuja, R.K.; Magnanti, T.L. \& Orlin, J.B. (1993). Network Flows. Prentice-Hall, Englewood Cliffs, New Jersey.

(2) Bell, M.H. (2003). Use of Game Theory to Measure Vulnerability of Networks. IEEE Trans. on Reliability, 52, 63-68.

(3) Boaventura Netto, P. (1996). Grafos: Teoria, Modelos e Algoritmos. Edgar Blücher, São Paulo.

(4) Caulkins, J.P.; Crawford, G. \& Reuter, P. (1993). Simulation of Adaptive Response: A Model of Drug Interdiction. Mathematical and Computer Modelling, 17, 37-52.

(5) Cormican, K.J.; Morton, D.P. \& Wood, R.K. (1998). Stochastic Network Interdiction. Operations Research, 46, 184-197.

(6) Danskin, J.M. (1962). Game Theory Model of Convoy Routing. Operations Research, 10, 774-785.

(7) Davis, M.D. (1973). Teoria dos Jogos. Cultrix e Editora da Universidade de São Paulo, São Paulo.

(8) Durbin, E.P. (1966). An Interdiction Model of Highway Transportation. Rand Corporation, RM-4945-PR.

(9) Ford, L.R. \& Fulkerson, D.R. (1954). Maximal Flow through a Network. Rand Corporation, RM 1400 e (1956). Canadian J. Math, 8, 399-404.

(10) Gass, S.I. (1969). Linear Programming. McGraw-Hill, New York.

(11) Grötschel, M.; Monma, C. \& Stoer, M. (1992). Computational Results with Cutting Plane Algorithms for Designing Communication Networks with Low Connectivity Constraints, Operations Research, 40, 309-330. 
(12) Hohzaki, R. \& Iida, K. (1996). Optimal Strategy of Route and Look for the Path Constrained Search Problem with Reward Criterion. European J. Operational Research, 100, 236-249.

(13) Israeli, E. (1999). System Interdiction and Defense. Tese de Doutorado - Naval Postgraduate School. Monterey, CA.

(14) Lima, K. \& Muniz, A. (2002). Detecção e Evasão em Redes, Experimentos Envolvendo um Problema de Médio Porte em uma Estrutura Radial - Departamento de Estatística, Universidade de Brasília, DF.

(15) Machado, H.V. \& Sinotti, R.L (2002). Confronto em Redes: Alguns Problemas em Aberto. First Brazilian Workshop of the Game Theory Society, São Paulo, 10 pgs.

(16) McMasters, A.W. \& Mustin, T.M. (1970). Optimal Interdiction of a Supply Network. Naval Research Logistics Quarterly, 17, 261-268.

(17) Mehdi, D. (1994). A Unified Approach to Network Survivability for Telegraphic Networks. IEEE Transactions on Communications, 42, 534-548.

(18) Schrijver, A. (2002). On the History of the Transportation and Maximum Flow Problems. Math Program, B91, 437-445.

(19) Sinotti, R.L. (2000). Confronto em Redes: Detecção e Evasão. Dissertação de Mestrado - Departamento de Estatística, Universidade de Brasília, DF.

(20) Steinrauf, R. (1991). A Network Interdiction Model. Dissertação de Mestrado - Naval Postgraduate School. Monterey, CA.

(21) Szwarcfiter, J.L. (1984). Grafos e Algoritmos Computacionais. Campus, Rio de Janeiro.

(22) Washburn, A.R. (1980). Search-Evasion Game in a Fixed Region. Operations Research, 28, 1290-1298.

(23) Washburn, A.R. (1983). Search for a Moving Target, the FAB Algorithm. Operations Research, 31, 739-751.

(24) Washburn, A.R. \& Wood, R.K. (1995). Two-Person Zero-Sum Games for Network Interdiction. Operations Research, 43, 243-251.

(25) Wollmer, R.D. (1964). Removing Arcs From a Network. Operations Research. 12, 934-940.

(26) Wollmer, R.D. (1970a). Algorithms for Targeting Strikes in a Lines-of-Communication Network. Operations Research, 18, 495-515.

(27) Wollmer, R.D. (1970b). Interception in a Network. Naval Research Logistic Quarterly, 17, 207-216.

(28) Wood, R.K. (1993). Deterministic Network Interdiction. Math. and Comput. Modeling, 17, $1-18$. 